\title{
Biological Activity and Chemical Composition of the Endophytic Fungus Fusarium sp. TP-G1 Obtained from the Root of Dendrobium officinale Kimura et Migo
}

\author{
Sanjun $\mathrm{Shi}^{\oplus}$, Yuanyuan $\mathrm{Li}^{\oplus}$, Yue Ming ${ }^{\oplus}$, Chenwen $\mathrm{Li}^{\oplus}$, Ziwei $\mathrm{Li}^{\oplus}$, \\ Jianhong Chen ${ }^{\odot}$ and Minghe Luo ${ }^{\oplus *}$
}

\author{
Department of Pharmacy, Institute of Surgery Research, Daping Hospital, Third Military Medical \\ University, Chongqing 400042, China
}

(Received December 30, 2017; Revised February 03, 2018; Accepted March 03,2018)

\begin{abstract}
Dendrobium officinable Kimura et Migo is a famous traditional herb medicine and is wildly used in folk as a tonic herb. Its endophytic fungi play prominent part in the function of D. officinable Kimura et Migo. In this study we first report the co-production of trichosetin (1), beauvericin (2), beauvericin A (3), enniatin B (4), enniatin H (5), enniatin I (6), enniatin MK1688 (7), fusaric acid (8) and dehydrofusaric acid (9) by the endophytic fungus Fusarium sp. TP-G1 deriving from the root of $D$. officinable kimura et Migo. These isolates' reported bioactivities and the tested biological activities in this paper consisted with the functions of $D$. officinable Kimura et Migo. We found the MICs value of compounds 1-7 against Staphylococcus aureus and MRSA showed nearly no difference, while compounds $\mathbf{8}-\mathbf{9}$ were first found to have the antimicrobial activities against Acinetobacter baumannii with a MIC value of $64 \mu \mathrm{g} / \mathrm{mL}$ and $128 \mu \mathrm{g} / \mathrm{mL}$ respectively. Compounds 2, 4, 5 and $\mathbf{6}$ were first found to show moderate anti-tumor activities against BL16F10; and compounds $\mathbf{4}$ and $\mathbf{5}$ were first found to show moderate anti-tumor activities against MCF-7. Furthermore, we report first here the isoleucine (Ile) replacement in beauvericin and enniatin will influence their antibacterial and brine shrimp lethality activities.
\end{abstract}

Keywords: Endophytic fungi; D. officinable Kimura et Migo; beauvericin; trichosetin; enniatin,;fusaric acid. () 2018 ACG Publications. All rights reserved.

\section{Introduction}

Endophytic fungi derived nature products are major resources to find drug leads [1-4]. D. officinable Kimura et Migo is considered to be the top of the famous immortal herb medicines, which has anti-tumor [5], anti-bacteria [6,7], antiviral [7], anti-hypertension activities [6,7] and so on. Therefore, it has been wildly used in folk as a tonic herb or health foods [5-7]. A piece of evidences showed that endophytic fungi of $D$. officinable Kimura et Migo may play prominent part in the function of its host by producing bioactive secondary metabolites [8]. However, the relationship between the secondary metabolites of the endophytic fungi and the function of its host remains uncertain due to the lack of specific researches on the secondary metabolites of endophytes derived from D. officinable Kimura et Migo. Fusarium, a large genus of filamentous fungi, are endophytic

* Corresponding author: E-Mail: $\underline{\operatorname{lmh} 353083 @ 126 . c o m}$; Phone:+86-23-68757098 
fungi commonly derived from plant, which can produce many famous bioactive compounds such as equisetin (anti-HIV and anti-bacterial activities) [9], coniosetin (anti-bacterial activity) [10] and fusarisetin A (inhibiting the tumor metastasis) [11], and so on. In light of this, the Fusarium genus fungi have become a rich and hot source for discovering drug leads.

Keep these in mind; we proceed in the isolation and bioactivities screening of endophytic fungi of $D$. officinable Kimura et Migo. In these efforts, we isolated and identified an endophytic fungus (Fusarium sp. TP-G1) from the root of D. officinable Kimura et Migo. Its fermentation extracts showed good shrimp lethality and antibacterial activities. The subsequent bioassay tracking isolation led to the simultaneous purification of trichosetin, beauvericin, beauvericin A, enniatin B, enniatin $\mathrm{H}$, enniatin I, enniatin MK1688, fusaric acid and dehydrofusaric acid. Furthermore, the biological activities assessment and structure-activity relationship of these isolates were also discussed in this paper.

\section{Materials and Methods}

\subsection{General}

Column chromatography were performed with 100-200 mesh silica gel of Jiangyou Silica Gel Development, Inc.. Mass spectra were operated on a Matrix-assisted laser desorption/ ionization time of flight mass spectrometry (Shimadzu). Preparative was performed on a QuikSep-50 system (H\&E High Quality \& Expert) using a YMC-Pack ODS-A column $(250 \times 10 \mathrm{~mm}, 5 \mu \mathrm{m})$. Analysis HPLC was operated with a 1260 infinity HPLC system (Agilent) equipment with a DAD detector. NMR spectra were operated on a DD2 600 spectrometer (Agilent) at $600 \mathrm{MHz}$ for ${ }^{1} \mathrm{H}$ and $150 \mathrm{MHz}$ for ${ }^{13} \mathrm{C}$.

\subsection{Isolation and Bioactivities Screening of Endophytic Fungi}

Fresh D.officinable kimura et Migo, which was identified by Professor Yunlong Li of Chongqing Academy of Chinese Materia Medica, was collected from specimen park of Chongqing Academy of Chinese Materia Medica in China on September 2015. A voucher specimen (No. 2015091516) is deposited at the Department of Pharmacy, Institute of Surgery Research, Daping Hospital, Third Military Medical University, China. The samples were put into polythene bags and tightly sealed at room temperature, then kept at $4{ }^{\circ} \mathrm{C}$ in the refrigerator after we return to the laboratory. We begin to isolate the fungal endophytes after $12 \mathrm{~h}$ of collection.

Before the isolation, every part of the collection of D. officinable kimura et Migo was surface sterilized. Briefly, they were first washed thoroughly with sterilized water for 4 minutes and then immersed in $70 \%$ ethanol for 1-2 min for tree times. After sterilizing, the plant was washed with sterilized water for 4 times again, and then it was separated into three parts (leaf, stem, root) on the sterilized dry absorbent paper. The sterilized and dried leaf, stem and root were cut into small pieces and placed on potato dextrose agar (PDA) media dishes. The emerged endophytic fungi of these tissues were sub-cultured on new PDA dishes until pure cultures were obtained. The isolated fungi were inoculated in flasks having $50 \mathrm{~mL}$ M-PDB medium (peel potato $20 \%$, dextrose $1 \%$, yeast exact power $0.3 \%$, mannitol $2 \%$, monosodium glutamate $0.5 \%$, bacterial peptone $0.5 \%$ ) and fermented at 28 ${ }^{\circ} \mathrm{C}$ with a rotary shaker $(200 \mathrm{rpm} / \mathrm{min})$ for 11 days, respectively. Then, they were extracted with 50 $\mathrm{mL}$ butanone and evaporated under reduced pressure to get crude extracts, respectively. The resulting crude extracts were pre-screened for their brine shrimp lethality activities and antimicrobial activities against Staphylococcus aureus (1312230073), Methicillin-resistant Staphylococcus aureus (MRSA) (131011097), Escherichia coli (100385570), Pseudomonas aeruginosa (202225634), Klebsiella pneumonia (202250163) and Acinetobacter baumannii (100069), which were provided by Department of Clinical Laboratory, Institute of Surgery Research, Daping Hospital, Third Military Medical University. The crude extracts, which showed antibacterial and brine shrimp lethality activities, were subject to HPLC for high-performance liquid chromatography-diode array detection (HPLC-DAD) screening (0-20 min: 0 to $85 \% \mathrm{~B} ; 20.1-25 \min 100 \% \mathrm{~B} ; 25.1-30 \mathrm{~min} 0 \% \mathrm{~B}$; A: $0.1 \% \mathrm{HOAc} / 15 \%$ $\mathrm{CH}_{3} \mathrm{CN}$ in $\mathrm{H}_{2} \mathrm{O}$, B: $0.1 \% \mathrm{HOAc} / 85 \% \mathrm{CH}_{3} \mathrm{CN}$ in $\mathrm{H}_{2} \mathrm{O}$; flow rate: $1.0 \mathrm{~mL} / \mathrm{min}$ ). 


\subsection{Identification of the EndophyticFfungi}

Pure culture in a dish of the endophytic fungus (TP-G1), which showed the most promising biological activities, was collected. Then, we amplified its $18 \mathrm{~S}$ rDNA sequence using a PCR method [28] and obtained a gene sequence of $532 \mathrm{bp}$. This sequence was submitted to GenBank to get the accession number (KY366174).

\subsection{Fermentation and Isolation}

A small amount of the fungus Fusarium sp. TP-G1 were inoculated into $250 \mathrm{~mL}$ Erlenmeyer flasks, each have $50 \mathrm{~mL}$ of M-PDB medium. These flask cultures were incubated on a rotary shaker of $200 \mathrm{rpm} / \mathrm{min}$ for 2 days at $28{ }^{\circ} \mathrm{C}$. Then they were transferred to $1000 \mathrm{~mL}$ Erlenmeyer flasks $(200 \mathrm{~mL}$ M-PDB medium) and were further incubated for another 11 days on the same condition above. The harvested $7.5 \mathrm{~L}$ cultures were centrifuged to get supernatant broths and mycelia. They were extracted three times with butanone (supernatant broths) and $\mathrm{MeOH}$ (mycelia) respectively to get two residues. Both of the residues of fermentation broths and mycelia were combined and subjected to a column chromatography (normal phase silica gel: 100-200 mesh) for isolation. It was eluted with a combination of solvent of $\mathrm{CH}_{2} \mathrm{Cl}_{2} / \mathrm{MeOH}(100 / 0,98 / 2,96 / 4,94 / 6,92 / 8,90 / 10,80 / 20,50 / 50 \mathrm{v} / \mathrm{v}$, each of $150 \mathrm{~mL}$ ) to obtain fractions Fr.1 to Fr.8. Fr.2 was further isolated by Sephadex LH-20 eluting with $\mathrm{MeOH}$ to give Fr.2-1-Fr.2-12. We combined Fr.2-(3-7) and further purified it with preparative HPLC using a YMC-Pack ODS-A column $(250 \times 10 \mathrm{~mm}, 5 \mu \mathrm{m})$, eluted with $80 \% \mathrm{CH}_{3} \mathrm{CN}$ over $35 \mathrm{~min}$ at $210 \mathrm{~nm}$ to get compounds $4(15.5 \mathrm{mg}), \mathbf{2}(1523 \mathrm{mg}), \mathbf{5}(20.2 \mathrm{mg}), \mathbf{3}(8.5 \mathrm{mg}), \mathbf{6}(16.4 \mathrm{mg}), \mathbf{7}(17.8 \mathrm{mg})$ with a retention time of $16.7 \mathrm{~min}, 17.7 \mathrm{~min}, 18.0 \mathrm{~min}, 18.5 \mathrm{~min}, 19.6 \mathrm{~min}, 24.3 \mathrm{~min}$, respectively. Fr.7 was also purified by preparative HPLC, eluting with a linear gradient of $75 \% \mathrm{CH}_{3} \mathrm{CN}$ to $100 \% \mathrm{CH}_{3} \mathrm{CN}$ over $30 \mathrm{~min}$ to obtain compound $1(18.5 \mathrm{mg})$ at $23.5 \mathrm{~min}$. After isolated with Sephadex LH-20, Fr.8 was further purified by preparative HPLC, eluting with $10 \% \mathrm{CH}_{3} \mathrm{CN}$ over $18 \mathrm{~min}$, to give $\mathbf{9}(14.1 \mathrm{mg})$, $8(68.5 \mathrm{mg})$ at $10.2 \mathrm{~min}, 13.5 \mathrm{~min}$.

\subsection{Antimicrobial Activity Assays}

Compounds 1-9 were further tested for MICs value using a broth dilution method [12]. Compounds 1-9 were serially diluted to concentrations of $0.0625-128 \mu \mathrm{g} / \mathrm{mL}$ by DMSO, and the MICs of compounds 1-9 were tested in triplicate. Cefuroxime was used as positive controls, while DMSO served as negative control.

\subsection{Brine shrimp Lethality and Anti-tumorAactivities Assays}

Brine shrimp lethality activities were performed as described [13] previously with modifications. Briefly, brine shrimp eggs were first hatched in artificial seawater for $36 \mathrm{~h}$. Then, every fifteen shrimps were added to one well of the 96-well plate. Finally, the tested crude extracts and compounds 1-9 were added to each well at tested concentrations respectively. The number of deaths of shrimps per well were recorded after $12 \mathrm{~h}$. The least concentration of the tested compound, which leads to the whole brine shrimp lethality in the well, was determined as the $\mathrm{LD}_{100}$ value of that compound. Compounds 1-9 were further tested for their anticancer activities against MCF-7 and B16F10 cell lines by MTT method [8]. Compounds 1-9 were serially diluted to concentrations of $0.625-20 \mu \mathrm{M}$, and all the data were tested in triplicate on a 96-well plate. 


\section{Results and Discussion}

\subsection{Identification and Pre-screening}

Eight endophytic fungi, including three fungi from the leaf, two fungi from the stem and three fungi from the root, were isolated from $D$. officinable Kimura et Migo. Among them, the crude fermentation extracts of the fungus TP-G1 (Figure 1) derived from the root of D. officinable Kimura et Migo, showed brine shrimp lethality activities and antibacterial activities against Staphylococcus aureus, MRSA and Acinetobacter baumannii. We identified this strain as Fusarium sp. TP-G1 by $18 \mathrm{~S}$ rDNA gene sequence analyses [28] and then subjected its crude extracts for HPLC-DAD screening (Figure 2).

\subsection{Isolation and structure elucidation}

HPLC-DAD guiding isolation resulted in the purification of compounds of 1-9 (Figure 3). The NMR data were summarized in Tables S1-8 separately.
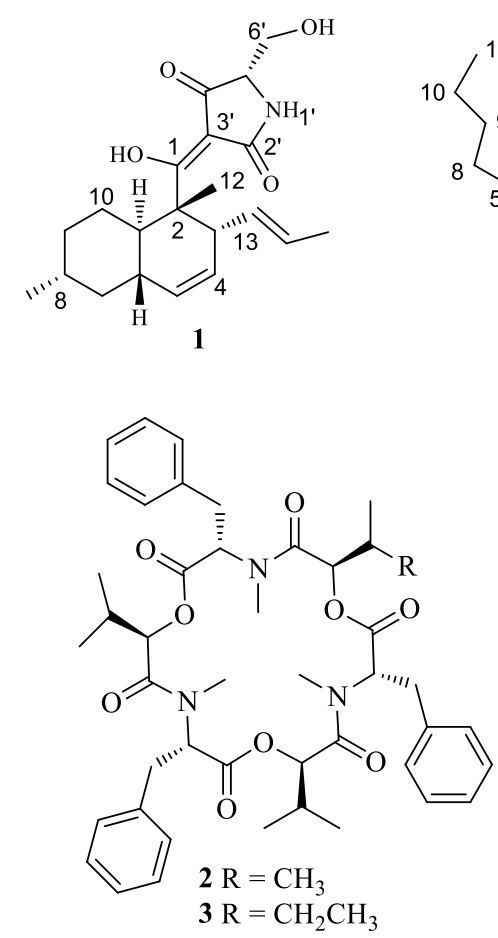<smiles>CCCCc1ccc(C(=O)O)nc1</smiles>

8

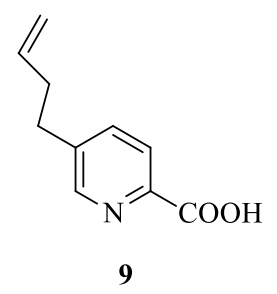

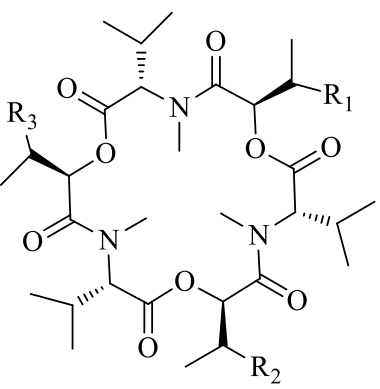

$4 \mathrm{R}_{1}=\mathrm{H}, \quad \mathrm{R}_{2}=\mathrm{H}, \quad \mathrm{R}_{3}=\mathrm{H}$ $5 \mathrm{R}_{1}=\mathrm{CH}_{2} \mathrm{CH}_{3}, \mathrm{R}_{2}=\mathrm{H}, \quad \mathrm{R}_{3}=\mathrm{H}$ $6 \mathrm{R}_{1}=\mathrm{CH}_{2} \mathrm{CH}_{3}, \mathrm{R}_{2}=\mathrm{CH}_{2} \mathrm{CH}_{3}, \mathrm{R}_{3}=\mathrm{H}$ $7 \mathrm{R}_{1}=\mathrm{CH}_{2} \mathrm{CH}_{3}, \mathrm{R}_{2}=\mathrm{CH}_{2} \mathrm{CH}_{3}, \mathrm{R}_{3}=\mathrm{CH}_{2} \mathrm{CH}_{3}$

Figure 3. Chemical structures of compounds 1-9.

The molecular formula of compound 1 was established to be $\mathrm{C}_{21} \mathrm{H}_{29} \mathrm{NO}_{4}$ by HRESIMS, which showed a molecular ion peak at $\left.\mathrm{m} / z 358.2009([\mathrm{M}-\mathrm{H}]]^{-}\right)$. The ${ }^{1} \mathrm{H}$ and ${ }^{13} \mathrm{C}$ NMR data (Tables S1) of compound $\mathbf{1}$ are very similarly to that of the known tetramic acid derivative trichosetin, which also have a molecular formula of $\mathrm{C}_{21} \mathrm{H}_{29} \mathrm{NO}_{4}$. Careful comparing of the NMR data between trichosetin and compound 1, lead to the identification of compound $\mathbf{1}$ as trichosetin [14].

Compound 2 was obtained as square crystals. The ${ }^{1} \mathrm{H}$ NMR spectrum of $\mathbf{2}$ was characterized by resonances consistent with two doublet methyl protons at $\delta_{\mathrm{H}} 0.38(3 \mathrm{H}, \mathrm{d}, J=6.6 \mathrm{~Hz})$ and $0.77(3 \mathrm{H}$, $\mathrm{d}, J=6 \mathrm{~Hz})$, one methylene at $\delta_{\mathrm{H}} 2.94(1 \mathrm{H}, \mathrm{t}, J=13.8 \mathrm{~Hz})$ and $3.36(1 \mathrm{H}, \mathrm{dd}, J=4.2,14.4 \mathrm{~Hz})$, one methine protons at $\delta_{\mathrm{H}} 1.96(1 \mathrm{H}, \mathrm{m})$, one $\mathrm{N}$-methyl resonance at $\delta_{\mathrm{H}} 2.99(3 \mathrm{H}, \mathrm{s})$, two nitrogen or oxygen-bearing methine protons at $\delta_{\mathrm{H}} 4.86(1 \mathrm{H}, \mathrm{d}, J=8.4 \mathrm{~Hz})$ and $5.54(1 \mathrm{H}, \mathrm{d}, J=7.8 \mathrm{~Hz})$, monosubstituted aromatic protons at $\delta_{\mathrm{H}} 7.15-7.25(5 \mathrm{H}, \mathrm{m})$. The ${ }^{13} \mathrm{C}$ NMR spectrum suggested the presence of fifteen carbon signals, including two carbonyls, three methines, one methylene, three 
methyls, and a benzene ring. Its molecular weight was determined to be $806.3\left([\mathrm{M}+\mathrm{Na}]^{+}\right)$by MS. These suggested that compound $\mathbf{2}$ was consists of three identical moieties. By comparing the NMR data (Table S2) with the data sets in published literature, compound $\mathbf{2}$ was identified as beauvericin [15].

In comparing of the NMR $\left({ }^{1} \mathrm{H}\right.$ and $\left.{ }^{13} \mathrm{C}\right)$ data of compound 2 with those of compounds $3-7$ (Tables S2 -S7), we found a great deal of similarity. Through careful inspection of their ${ }^{1} \mathrm{H}$ and ${ }^{13} \mathrm{C}$ NMR spectral data with those reported in literature, compounds 3-7 were identified as beauvericin A (3) [16], enniatin B (4) [17], enniatin H (5) [18], enniatin I (6) [18], enniatin MK1688 (7) [18] respectively.

The ${ }^{1} \mathrm{H}$ NMR spectrum of 8 was consisted of one doublet methyl protons at $\delta_{\mathrm{H}} 0.86(3 \mathrm{H}, \mathrm{d}, J=$ $6.6 \mathrm{~Hz})$, three methylene at $\delta_{\mathrm{H}} 1.30(2 \mathrm{H}, \mathrm{m}), 1.58(2 \mathrm{H}, \mathrm{m}), 2.68(2 \mathrm{H}, \mathrm{t}, J=7.2 \mathrm{~Hz})$, three pyridine protons at $\delta_{\mathrm{H}} 7.73(1 \mathrm{H}, \mathrm{d}, J=7.2), 8.16(1 \mathrm{H}, \mathrm{s}), 8.74(1 \mathrm{H}, \mathrm{s})$, one $\mathrm{COOH}$ proton at $\delta_{\mathrm{H}} 13.16(1 \mathrm{H}, \mathrm{s})$. The ${ }^{13} \mathrm{C}$ NMR spectrum unveiled signals corresponding to one carbonyl at $\delta_{\mathrm{C}} 165.5$, three aromatic heterocycte methine carbons, and two aromatic heterocycte non-protonated carbons, three methylenes, one methyl. Its molecular weight was obtained by MS, which showed a $[\mathrm{M}+\mathrm{H}]^{+}$ion at $\mathrm{m} / \mathrm{z} 180.1$. Finally, compound $\mathbf{8}$ was determined as a fusaric acid by careful comparing with reported NMR data of literature. ${ }^{1} \mathrm{H}$ spectroscopic data for 9 closely resembled those of $\mathbf{8}$ (Table S8) with the exception of the loss of one methylene at $\delta_{\mathrm{H}} 0.86(3 \mathrm{H}, \mathrm{t}, 7.2)$ and one methylene at $\delta_{\mathrm{H}} 1.30(2 \mathrm{H}, \mathrm{m})$, and the presence of three additional olefinic protons $\delta_{\mathrm{H}} 4.99(2 \mathrm{H}, \mathrm{d}, 12.6)$ and $\delta_{\mathrm{H}} 5.78(1 \mathrm{H}, \mathrm{m})$. In agreement with this, the ${ }^{13} \mathrm{C}$ NMR spectroscopic data analysis showed the ${ }^{13} \mathrm{C}$ NMR chemical shifts were changed from $\delta_{\mathrm{C}} 22.3, \delta_{\mathrm{C}} 13.8$ to $\delta_{\mathrm{C}} 138.8, \delta_{\mathrm{C}} 116.3$ (Table $\mathbf{S 8}$ ). Thus, compound $\mathbf{9}$ was identified as dehydrofusaric acid, which agreed with the NMR data of reported in the literature [19].

The isolation of endophytic fungi of D. officinable Kimura et Migo were studied previously, but little had been done to the isolation and identification procedures of the endophytic fungi and associated bioactive secondary metabolites of $D$. officinable Kimura et Migo. This is the first report of simultaneous isolation of trichosetin, beauvericin, beauvericin A, enniatin B, enniatin $\mathrm{H}$, enniatin I, enniatin MK1688, fusaric acid and dehydrofusaric acid from the endophytic fungus (Fusarium sp. TPG1) of D. officinable Kimura et Migo.

\subsection{Antimicrobial Activities}

According to the antibacterial activities results of the crude fermentation extracts of Fusarium sp. TP-G1, the purified compounds 1-9 were further tested MICs value against Staphylococcus aureus, MRSA and Acinetobacter baumannii.

The results were summarized in Table $\mathbf{1}$. It showed that compounds 1-7 were responsible for the antimicrobial activity against Staphylococcus aureus and MRSA; while compounds $\mathbf{8}$ and $\mathbf{9}$ were in charge of the antibacterial activities against Acinetobacter baumannii.

Table 1. Antimicrobial activities (MICs, $\mu \mathrm{g} / \mathrm{mL}$ ) of compounds 1-9

\begin{tabular}{lccc}
\hline Compounds & Staphylococcus aureus & MRSA & Acinetobacter baumannii \\
\hline Trichosetin (1) & 2 & 2 & $>128$ \\
Beauvericin (2) & 4 & 4 & $>128$ \\
Beauvericin A (3) & 2 & 2 & $>128$ \\
Enniatin B (4) & 128 & 128 & $>128$ \\
Enniatin H (5) & 32 & 32 & $>128$ \\
Enniatin I (6) & 8 & 16 & $>128$ \\
Enniatin MK1688(7) & 4 & 8 & $>128$ \\
Fusaric acid (8) & $>128$ & $>128$ & 64 \\
Dehydrofusaric acid (9) & $>128$ & $>128$ & 128 \\
Cefuroxime & 0.125 & $>128$ & $>128$ \\
\hline
\end{tabular}

These results showed that: except enniatin B (4) exhibited a MIC value of $128 \mu \mathrm{g} / \mathrm{mL}$, which had been reported to have activities against Staphylococcus aureus CECT 976, but not Staphylococcus aureus CECT 240 [20], both isolated beauvericins and enniatins showed good antibacterial activities 
against Staphylococcus aureus and MRSA, and their MICs value against Staphylococcus aureus and MRSA showed no difference. We also first found compounds 8 and 9 have an MIC value of $64 \mu \mathrm{g} / \mathrm{mL}$ and $128 \mu \mathrm{g} / \mathrm{mL}$ against Acinetobacter baumannii respectively.

\subsection{Brine shrimp lethality and anti-tumor activities}

Brine shrimp lethality activities have good relationship with the cell proliferation inhibitory activity [13]. So according to the $\mathrm{LD}_{100}$ results of brine shrimp toxic activities, we also further tested the $\mathrm{IC}_{50}$ against MCF-7 and BL16F10 cell lines of compounds 1-9. The results were summarized in Table 2. Although compounds $\mathbf{2}$ and $\mathbf{6}$ had been reported to have antitumor activities against MCF-7 with $\mathrm{IC}_{50}$ value of $1.4 \mu \mathrm{g} / \mathrm{mL}(1.79 \mu \mathrm{M})$ [21] and $1.0 \mu \mathrm{M}$ [22], but in this study we only found it had an $\mathrm{IC}_{50}$ value of $12.4 \mu \mathrm{M}$ and $10.27 \mu \mathrm{M}$ respectively. We also first report here the cytotoxic activities of compounds $2, \mathbf{4}, \mathbf{5}, \mathbf{6}$ against BL16F10 and the anticancer activities of compounds $\mathbf{4}, \mathbf{5}$ against MCF-7.

Table 2. Anti-tumor $\left(\mathrm{IC}_{50}\right.$ values, $\left.\mu \mathrm{M}\right)$ and brine shrimp lethality $\left(\mathrm{LD}_{100}\right.$ values, $\left.\mu \mathrm{g} / \mathrm{mL}\right)$ ) activity assays of compounds $\mathbf{1}^{-9}$

\begin{tabular}{lccc}
\hline Compounds & $\begin{array}{c}\text { MCF-7 } \\
\left(\text { IC }_{\mathbf{5 0}}\right)\end{array}$ & B16F10 $\left(\mathbf{I C}_{\mathbf{5 0}}\right)$ & $\begin{array}{c}\text { LD }_{\mathbf{1 0 0}} \text { for brine } \\
\text { shrimp }\end{array}$ \\
\hline Trichosetin (1) & $>20$ & $>20$ & 64 \\
Beauvericin (2) & 12.4 & 15.2 & 64 \\
Beauvericin A (3) & $>20$ & $>20$ & 32 \\
Enniatin B (4) & 12.22 & 10.64 & 8 \\
Enniatin H (5) & 11.46 & 8.39 & 16 \\
Enniatin I (6) & 10.27 & 9.46 & 16 \\
Enniatin MK1688(7) & $>20$ & $>20$ & 32 \\
Fusaric acid (8) & $>20$ & $>20$ & $>128$ \\
Dehydrofusaric acid (9) & $>20$ & $>20$ & $>128$ \\
Doxorubicin & 6.2 & 1.7 & not tested \\
\hline
\end{tabular}

Based on the antibacterial and shrimp lethality activities results (Table $\mathbf{1}$ and Table 2), we found some structure-activity relationship between the beauvericins and enniatins in their brine shrimp lethality activities and antibacterial activities. Results suggested that: the Val replaced by Ile in the structure of beauvericin and enniatin can enhance their antibacterial activities; while for the brine shrimp lethality activities, only enniatins had structure-activity relationship on the other way round. As we known, beauvericins and enniatins have good biological activities, so the structure-activity relationship may help to modify the structures for clinical application.

Although compounds 1-9 were all well known mycotoxins and have been reported of diverse biological activities, such as: HIV-1 integrase inhibition activities from beauvericins and enniatins [23]; antiplasmodia and antimycobacterial activities from beauvericins and enniatins [21,22]; antibacterial activities from trichosetin, beauvericins and enniatins [14, 21, 22]; antitumor activities from beauvericins and enniatins [21, 22]; anti-hypertension activities from fusaric acid and dehydrofusaric acid [24, 25]; acyl-CoA: cholesterol acyltransferase (ACAT) inhibition activities from beauvericins and enniatins $[14,22,26]$; insecticidal activities and antiviral activities from beauvericins [21]. But in these study we not only first simultaneously isolated compounds $\mathbf{1}-\mathbf{9}$ from endophytic fungus (Fusarium sp. TP-G1) of D. officinable Kimura et Migo, but also systemly evaluated their bioactivities. Among them, the shrimp lethality and anticancer activities against MCF-7 of compound $\mathbf{1}$ and compounds 3-9; the anti-tumor activities against BL16F10 and the antibacterial activities against Acinetobacter baumannii of compounds 1-9; and the anti-MRSA activities of compounds 3-9 were first reported here. 


\section{Conclusions}

There are researchers reported on the co-production of enniatins with beauvericin by Fusarium species [27]. However, there have been no previous reports related to the co-production of trichosetin, fusaric acids, anniatins and beauvericins by Fusarium species. More importantly this is the first report of an endophytic fungus of $D$. officinable Kimura et Migo being able to produce trichosetin, beauvericin, beauvericin A, enniatin B, enniatin H, enniatin I, enniatin MK1688, fusaric acid and 9,10dehydrofusaric acid simultaneously.

D. officinable Kimura et Migo, which know as the top of the immortal herb medicines, has anti-tumor, anti-bacteria, antiviral, anti-hypertension activities and so on; and were wildly used in folk as a tonic herb or health foods. The previously reported diverse biological activities and the reported biological activities of compounds 1-9 in this paper consisted with the functions of D. officinable kimura et Migo, which indicated that the endophytic fungi not only assist the host to withstand stressful conditions but also may play a role in the functions of $D$. officinable kimura et Migo. This means the function of $D$. officinable Kimura et Migo may have relationship with its endophytic fungi. In light of fusafungine (a mixture of enniatins, which was got from the fermentation of a stain of Fusarium) was a topical treatment for upper respiratory tract infections [22]. Fusarium sp. TP-G1, which was capable of producing so many kinds of bioactive compounds, such as: trichosetin, beauvericin, enniatins, fusaric acid and dehydrofusaric acid, also has great potentialities to develop clinical medicine.

\section{Acknowledgments.}

This study was supported by Chongqing Municipal Bureau of Health Science and technology of traditional Chinese medicine project (ZY20132062).

\section{Supporting Information}

\section{ORCID}

Supporting information accompanies this paper on http://www.acgpubs.org/RNP

Sanjun Shi: 0000-0002-0380-3517

Yuanyuan Li: $\underline{0000-0001-7772-8487}$

Yue Ming: 0000-0003-4643-7817

Chenwen Li: 0000-0002-1923-139X

Ziwei Li: 0000-0002-5153-4227

Jianhong Chen: 0000-0003-2514-9840

Minghe Luo: 0000-0002-2565-9237

\section{References}

[1] A. Ganesan (2008). The impact of natural products upon modern drug discovery, Curr. Opin. Chem. Biol. 12(3), 306-317.

[2] A.L. Harvey (2008). Natural products in drug discovery, Drug. Discov. Today. 13(19-20), 894-901.

[3] H. Nisa, A.N. Kamili, I.A. Nawchoo, S. Shafi, N. Shameem, and S.A. Bandh (2015). Fungal endophytes as prolific source of phytochemicals and other bioactive natural products: A review, Microb. Pathog. 82, 50-59.

[4] T.S. Suryanarayanan (2013). Endophyte research: going beyond isolation and metabolite documentation, Fungal. Ecol. 6(6), 561-568.

[5] G.Y. Lv, M.Q. Yan, and S.H. Chen (2013). Review of pharmacological activities of Dendrobium officinale based on traditional functions, China Journal of Chinese Materia Medica 38(4), 489-493.

[6] J.A. Teixeira da Silva, and T.B. Ng (2017). The medicinal and pharmaceutical importance of Dendrobium species, Appl. Microbiol. Biotechnol. 101(6), 2227-2239.

[7] H. Tang, T. Zhao, Y. Sheng, T. Zheng, L. Fu, and Y. Zhang (2017). Dendrobium officinale Kimura et Migo: A Review on Its Ethnopharmacology, Phytochemistry, Pharmacology, and Industrialization, Evid. Based. Complement. Alternat. Med. 2017, 7436259.

[8] L.S. Wu, M. Jia, L. Chen, B. Zhu, H.X. Dong, J.P. Si, W. Peng, and T. Han (2015). Cytotoxic and Antifungal Constituents Isolated from the Metabolites of Endophytic Fungus DO14 from Dendrobium 
officinale, Molecules 21(1), E14.

[9] Y.C. Jeong, and M.G. Moloney (2015). Equisetin, reutericyclin and streptolodygin as natural product lead structures for novel antibiotic libraries, Future. Med. Chem. 7(14), 1861-1877.

[10] M.P. Segeth, A. Bonnefoy, M. Brönstrup, M. Knauf, D. Schummer, L. Toti, L. Vértesy, M.C. WetzelRaynal, J. Wink, and G. Seibert (2003). Coniosetin, a novel tetramic acid antibiotic from Coniochaeta ellipsoidea DSM 13856, J. Antibiot. 56(2), 114-122.

[11] J.H. Jang, Y. Asami, J.P. Jang, S.O. Kim, D.O. Moon, K.S. Shin, D. Hashizume, M. Muroi, T. Saito, H. Oh, B.Y. Kim, H. Osada, and J.S. Ahn (2011). Fusarisetin A, an acinar morphogenesis inhibitor from a soil fungus, Fusarium sp. FN080326, J. Am. Chem. Soc. 133(18), 6865-6867.

[12] X. Zhou, H.B. Huang, J. Li, Y.X. Song, R.W. Jiang, J. Liu, S. Zhang, Y. Hua, and J.H. Ju (2014). New anti-infective cycloheptadepsipeptide congeners and absolute stereochemistry from the deep sea-derived Streptomyces drozdowiczii SCSIO 10141, Tetrahedron 70, 7795-7801.

[13] S.S. Patel, and M.N. Zaveri (2011). Cytotoxic activity to find bioactive compound from Justicia gendarussa using brine shrimp lethality assay, Asian Journal of Traditional Medicines 7(3), 102108.

[14] J. Inokoshi, N. Shigeta, T. Fukuda, R. Uchida, K. Nonaka, R. Masuma, and H. Tomoda (2013). Epitrichosetin, a new undecaprenyl pyrophosphate synthase inhibitor, produced by Fusarium oxysporum FKI-4553, J. Antibiot. 66(9), 549-554.

[15] A.R.B. Ola, A.H. Aly, W.H. Lin, V. Wray, and A. Debbab (2014). Structural revision and absolute configuration of lateritin, Tetrahedron. Lett. 55(45), 6184-6187.

[16] S. Gupta, C. Montllor, and Y.S. Hwang (1995). Isolation of novel beauvericin analogues from the fungus Beauveria bassiana, J. Nat. Prod. 58(5), 733-738.

[17] V. Cuomo, A. Randazzo, G. Meca, A. Moretti, A. Cascone, O. Eriksson, E. Novellino, and A. Ritieni (2013). Production of enniatins A, A1, B, B1, B4, J1 by Fusarium tricinctum in solid corn culture: structural analysis and effects on mitochondrial respiration, Food. Chem. 140(4), 784-793.

[18] C. Nilanonta, M. Isaka, R. Chanphen, N. Thong-Orn, M. Tanticharoen, and Y. Thebtaranonth (2003). Unusual enniatins produced by the insect pathogenic fungus Verticillium hemipterigenum: Isolation and studies on precursor-directed biosynthesis, Tetrahedron 59(7), 1015-1020.

[19] H.R. Burmeister, M.D. Grove, R.E. Peterson, D. Weisleder, and R.D. Plattner (1985). Isolation and characterization of two new fusaric acid analogs from Fusarium moniliforme NRRL 13,163, Appl Environ Microbiol 50(2), 311-314.

[20] G. Meca, I. Sospedra, M.A. Valero, J. Mañes, G. Font, and M.J. Ruiz (2011), Antibacterial activity of the enniatin B, produced by Fusarium tricinctum in liquid culture, and cytotoxic effects on Caco-2 cells, Toxicol. Mech. Methods. 21(7), 503-512.

[21] Q.G. Wang, and L.J. Xu (2012). Beauvericin, a Bioactive Compound Produced by Fungi: A Short Review, Molecules 17(3), 2367-2377.

[22] A.A. Sy-Cordero, C.J. Pearce, and N.H. Oberlies (2012). Revisiting the enniatins: a review of their isolation, biosynthesis, structure determination and biological activities, J. Antibiot. 65(11), 541-549.

[23] C.G. Shin, D.G. An, H.H. Song, and C. Lee (2009). Beauvericin and enniatins H, I and MK1688 are new potent inhibitors of human immunodeficiency virus type-1 integrase, J. Antibiot. 62(12), 687690.

[24] H. Hidaka, T. Nagatsu, K. Takeya, T. Takeuchi, and H. Suda (1969). Fusaric acid, a hypotensive agent produced by fungi, J. Antibiot. 22(5), 228-230.

[25] H. Wang, and T.B. Ng (1999). Pharmacological activities of fusaric acid (5-butylpicolinic acid), Life. Sci. 65(9), 849-856.

[26] R. Lemmens-Gruber, M.R. Kamyar, and R. Dornetshuber (2009). Cyclodepsipeptides-potential drugs and lead compounds in the drug development process, Curr. Med. Chem. 16(9), 1122-1137.

[27] H.H. Song, J.H. Ahn, Y.H. Lim, and C. Lee (2006). Analysis of beauvericin and unusual enniatins co-produced by Fusarium oxysporum FB1501 (KFCC 11363P), J. Microbiol. Biotechn. 16(7), 11111119.

[28] M. Luo, Z. Cui, H. Huang, X. Song, A. Sun, Y. Dang, L. Lu, and J. Ju (2017). Amino Acid Conjugated Anthraquinones from the Marine-Derived Fungus Penicillium sp. SCSIO sof101, J. Nat. Prod. 80 (5), 1668-1673.

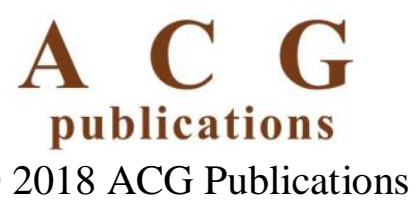

